\title{
Matching functions between loudness and ten other continua'
}

Cross-modality matches have been made between loudness and ten other perceptual continua. The matching functions are all power functions. When the exponent values of the matching functions are divided by the exponent values previously determined for the various continua, the quotients predict values for the loudness exponent. A tentative consensus suggests that the loudness exponent may be about 0.64 .

The well documented finding that the loudness of a $1000-\mathrm{Hz}$ tone increases as a power function of sound pressure is perhaps by itself no more noteworthy than any isolated empirical relation. What may catalyze our interest, however, is the additional fact that every sensory continuum exhibits the same invariance: equal stimulus ratios produce equal sensation ratios. The sweep of that principle has opened a fertile prospect. We can verify the form and the exponent of the loudness function by matching loudnesses to values on each of the other sensory continua. Matches between loudness and ten other continua have in fact been completed, and the purpose here is to review the outcome. The results are generally consistent with the quantitative relation between loudness $\mathrm{L}$ and sound pressure $p$ given by the equation

$$
L=k\left(p-p_{0}\right)^{0.6}
$$

where $k$ is a constant that depends upon units and $p_{o}$ is the threshold value.
Despite differences in the experimental procedures used in the various matching experiments, the value of the exponent 0.6 was shown to be approximately correct, but there appears to be a growing consensus that the second significant figure may be decidedly greater than zero.

The ability of a matching procedure to verify an exponent rests on the following basis. If, given an appropriate choice of units, two sense modalities are governed by the power laws

$$
\psi_{1}=\phi_{1}^{\alpha}
$$

and

$$
\psi_{2}=\phi_{2} \beta
$$

and if the sensation values $\psi_{1}$ and $\psi_{2}$ are equated by cross-modality matches at a number of different levels, then the result will be an equal-sensation function. This function will determine a relation between the stimuli of the two sense modalities of the form

$$
\phi_{1}^{\alpha}=\phi_{2} \beta
$$

or in terms of logarithms

$$
\log \phi_{1}=(\beta / a) \log \phi_{2}
$$

In other words, in log-log, or decibel, coordinates the equal-sensation function becomes a straight line whose slope is $\beta / \alpha$, the ratio of the two original expo-

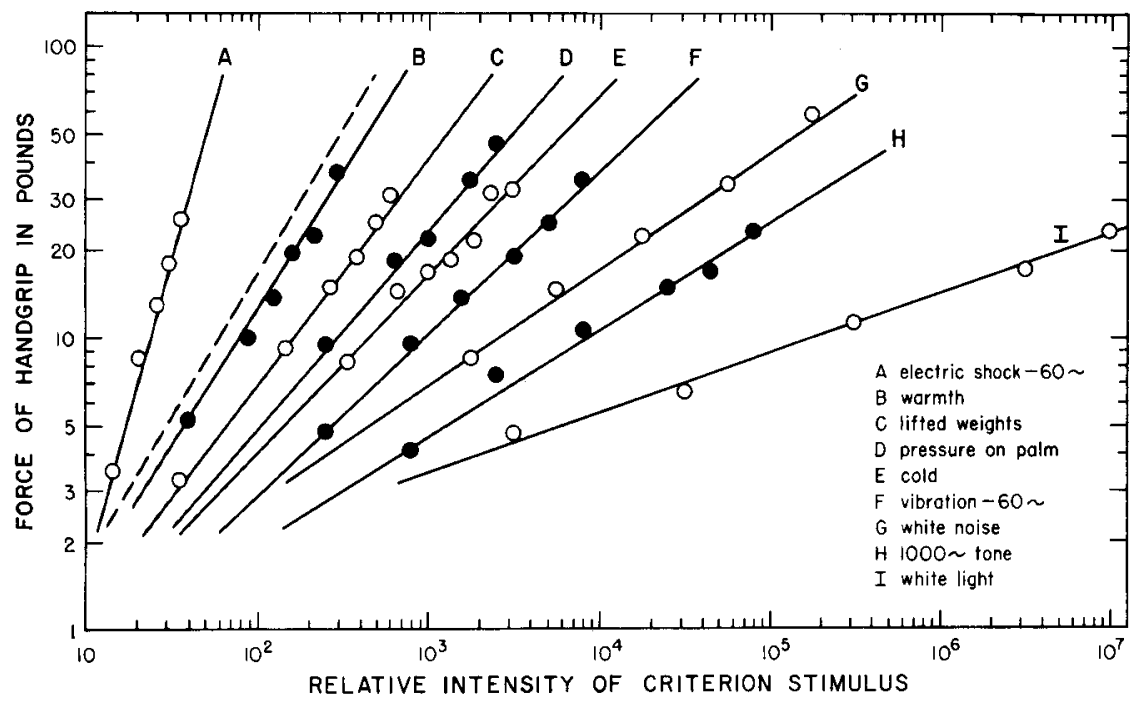

Fig. 1. Equal-sensation functions obtained by matching force of handgrip to various criterion stimuli. Each point stands for the median force exerted by 10 or more observers to match the apparent intensity of a criterion stimulus. The relative position of a function along the abscissa is arbitrary. The dashed line shows a slope of 1.0 in these coordinates. 
nents. Thus if the exponent for loudness is 0.6 and the exponent for apparent force of handgrip is 1.7 , then, when observers squeeze a dynamometer to match a series of loudnesses, the matching function should have a $\log -\log$ slope (exponent) of $0.6 / 1.7$ or 0.35 . That, in fact, was the value of the exponent actually obtained, as shown in Fig. 1.

The slopes of the power functions in Fig. 1 give a direct indication of the wide range of exponents that govern the various sense modalities. The functions are derived from several different experiments, all of which were conducted by J. C. Stevens and his collaborators.

In a large number of other experiments, crossmodality matches have been made with loudness rather than handgrip, and from these matching experiments a set of power functions can be plotted relating loudness to each of the other perceptual continua. Ten such matching functions are shown in Fig. 2. Each point represents the geometric mean of matches made by a group of observers, usually ten. Details concerning the experiments can be found in the articles referenced below. The sounds used were bands of noise between the nominal frequency limits specified in Table 1. A pure tone was used when the observers matched loudness to a series of numbers designated by the experimenter.

Most of the functions in Fig. 2 show the results of varying loudness to match each of a series of magnitudes on some other continuum. In three instances, handgrip, vocal effort, and length, fixed sound pressure levels were set and the observer varied the other stimulus to match the loudness. For four continua, electric current, vibration, number, and brightness, a balanced procedure was carried out in which loudness served as the variable in one part of the experiment and as the fixed or criterion value in another part. The results of the balanced experiments in which matching was carried out in both directions exhibit the ubiquitous regression effect: in coordinates like those shown in Fig. 2 the function is slightly steeper when the other continuum is matched to loudness. The amount of regression, measured by the difference in the two slopes, was rather small for electric current and for vibration. The more difficult task of matching loudness to brightness and brightness to loudness showed a larger regression effect, as did also the matching of loudness to numbers and numbers to loudness. It appears that the angle between the two regression lines produced by a balanced experiment may provide a direct indication of the amount of noise, uncertainty, or difficulty in the matching process. Unfortunately some kinds of stimuli cannot be easily placed under the observer's control; consequently the amount of regression cannot always be measured.

\section{Exponents}

In a previous study (J. C. Stevens, Mack, \& S. S. Stevens, 1960) it was shown that the functions in Fig. 1 form a consistent family in the sense that the slopes (exponents) agree with the values independently predicted. The largest disagreement between the slope predicted from the results obtained by matching against the number continuum and the slope obtained by matching against handgrip squeezes involved white noise (predicted 0.35 , obtained 0.41 ). The agreement is improved, however, if the exponent for loudness is assumed to be slightly larger than 0.6 , which, in fact, is what the evidence in Fig. 2 suggests.

The slopes of the matching functions in the log-log coordinates of Fig. 2 are entered in Table 1, together with the exponents obtained in experiments in which matching was made by one or another form of numerical estimation. The ratio between the exponent re number and the $\log -\log$ slope obtained by matching a continuum against loudness gives a predicted exponent for the loudness function, as shown in Table 1. Ideally, all the predicted exponents should have the same value. Except when the exponent was determined by squeezing rubber samples (hardness), the predicted exponents fall between

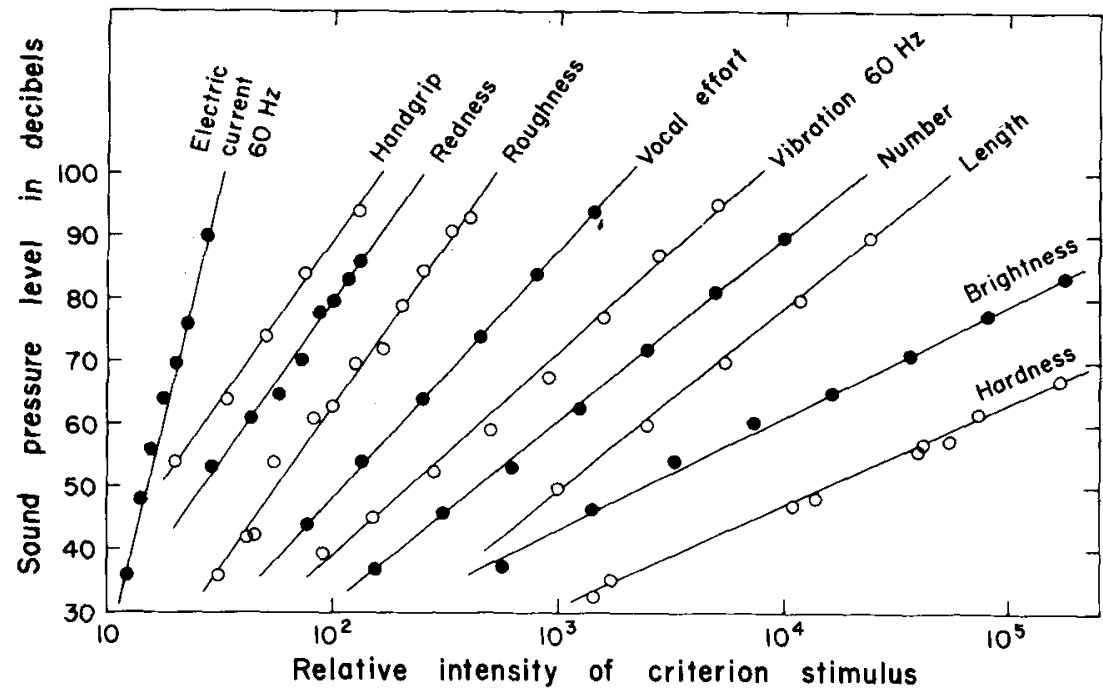

Fig. 2. Equal-sensation functions obtained by matches between loudness and various criterion stimuli. The relative positions of the functions are arbitrary, but the slopes are those determined by the data. 
0.55 and 0.75 with an average value of 0.64 .

The purpose in averaging the loudness exponents in Table 1 is not to try to specify the second decimal place at the present time. Rather it is to suggest that the convergence of the evidence is sufficiently promising to sustain the hope that a more definitive value may be forthcoming in the future. The functions pictured in Fig. 2 and the exponents listed in Table 1 serve only as examples. They do not constitute an exhaustive survey.

\section{The Power Function}

Two important features characterize the families of functions in Figs. 1 and 2. All the matching functions are power functions, and, with the exception of the function for hardness, there is a transitivity among the slopes (exponents) such that any two can be used to predict a third. For example, if we have matched handgrip to loudness and loudness to vibration, we can then predict an interesting sequence of behavior, namely, how hard observers will squeezea hand dynamometer in order to match the apparent force of their squeezes to the apparent intensities of various vibration amplitudes applied to a finger.

In their excellent review of scaling, Ekman \& Sjoberg (1965) point out that, despite the massive evidence for the psychophysical power law, questions continue to be raised largely because the initial developments rested mainly on methods that involved numerical estimations. In retrospect, one is tempted to wish that numerical procedures like magnitude estimation had never been invented, for much semantic and metaphysical argument has hinged on issues raised by the observer's use of numbers. Let us imagine that the function relating loudness to number had been deleted from Fig. 2 and that no numerical estimating had been or ever would be per- formed. What substantive questions about the over-all operating characteristics of the sensory continua would we then be unable to answer? Forbidden to use number, we could refer the slopes (exponents) in Fig. 2 to some other reference continuum, such as length of lines. For in the business of measurement there are no absolute values; there are only relative values. Referred to length, then, the exponent for each continuum could be calculated on the assumption that the exponent for apparent length is 1.0 , which appears to be a plausible assumption.

Our ability to specify the power functions relative to length instead of number seems to contradict the statement of Ekman and Sjoberg that "the power law is always verified by direct ratio estimation methods, and only by these methods." With no resort to estimation methods it has been shown in Fig. 2 that the over-all transfer functions of several sense modalities are related to one another as a family of power functions. Furthermore, in order to demonstrate the powerfunction relations by means of cross-modality matching, we need only assume that the observer can adjust one stimulus (measured on a ratio scale) to make it appear as strong as another. No other postulate is needed.

Do the families of power functions in Figs. 1 and 2 dispose of Fechner's logarithmic law? Time alone will answer that question. In the meantime, although the families of power functions generated by the procedure of cross-modality matching have seemed reasonably convincing, they have apparently failed to satisfy at least two kinds of Fechnerians: those who cling to Fechner's postulate that equal stimulus ratios produce equal sensation differences (e.g., that jnds are subjectively equal), and those who are willing to postulate

Table I. The first column shows the exponents determined by numerical estimation (matching to number). The second column shows the slopes of the functions in Fig. 2 . The ratio of the values in the first two columns predicts an exponent for the loudness function, as listed in the third column. The exponent for brightness is stated as 0.66 instead of the usual 0.33 in order to make it comparable to the loudness exponent, which is here given in terms of an amplitude rather than an energy measure.

\begin{tabular}{|c|c|c|c|c|c|}
\hline & $\begin{array}{l}\text { Exponent } \\
\text { re number }\end{array}$ & $\begin{array}{l}\text { Motch } \\
\text { slope }\end{array}$ & $\begin{array}{l}\text { Predicted } \\
\text { exponent } \\
\text { for loudness }\end{array}$ & $\begin{array}{l}\text { Bandwidth } \\
\text { of sound }\end{array}$ & Reference \\
\hline $\begin{array}{l}\text { Electric current }(60 \mathrm{~Hz}) \\
\text { through fingers }\end{array}$ & 4.5 & 7.5 & 0.60 & $100-500 \mathrm{~Hz}$ & Stevens (1959a) \\
\hline Force of handgrip & 1.7 & 2.5 & 0.68 & $100-6600$ & $\begin{array}{l}\text { J. C. Stevens, Mack, \& } \\
\text { S. S. Stevens }(1960)\end{array}$ \\
\hline Redness (saturation) & 1.7 & 2.6 & 0.65 & $100-3000$ & $\begin{array}{l}\text { Panek \& Stevens (in } \\
\text { press) }\end{array}$ \\
\hline $\begin{array}{l}\text { Tactile roughness of } \\
\text { emery cloth }\end{array}$ & 1.5 & 2.6 & 0.58 & $500-5000$ & Stevens \& Harris (1962) \\
\hline Vocal effort & 1.1 & 2.0 & 0.55 & $100-2000$ & $\begin{array}{l}\text { Lane, Catania, \& } \\
\text { Stevens (1961) }\end{array}$ \\
\hline Vibration on finger & 0.96 & 1.6 & 0.60 & $100-500$ & Stevens (1959b) \\
\hline Number & 1.0 & 1.45 & 0.69 & $\begin{array}{l}1000 \mathrm{~Hz} \\
\text { tone }\end{array}$ & Stevens \& Guirao (1962) \\
\hline Length of line & 1.0 & 1.45 & 0.69 & $500-5000$ & Stevens \& Guirao (1963) \\
\hline Visual brightness & 0.66 & 0.88 & 0.75 & $75-4800$ & $\begin{array}{l}\text { J. C. Stevens \& Marks } \\
\text { (1965) }\end{array}$ \\
\hline $\begin{array}{l}\text { Hardness of rubber } \\
\text { by squeezing }\end{array}$ & 0.8 & 0.81 & 0.99 & $500-5000$ & Harper \& Stevens (1964) \\
\hline
\end{tabular}


that a logarithmic function intervenes somewhere between stimulus excitation and some inner subjective state. Both kinds of postulates have a certain seductive quality, but, as Bertrand Russell (1920) said, "The method of 'postulating' what we want has many advantages; they are the same as the advantages of theft over honest toil."

\section{References}

Ekman, G., \& Sjöberg, L. Scaling. Ann. Rev. Psychol., 1965, 16, 451-474.

Harper, R., \& Stevens, S. S. Subjective hardness of compliant materials. Quart. J. exp. Psychol., 1964, 16, 204-215.

Lane, H. L., Catania, A. C., \& Stevens, S. S. Voice level: Autophonic scale, perceived loudness, and effects of sidetone. $J$. Acoust. Soc. Amer., 1961, 33, 160-167.

Panek, D. W., \& Stevens, S. S. Saturation of red: A prothetic continuum. Percept. Psychophys., in press.

Russell, B. Introduction to mathematical philosophy. (2nd. ed.) New York: Macmillan, 1920.
Stevens, J. C., Mack, J. D., \& Stevens, S. S. Growth of sensation on seven continua as measured by force of handgrip. J. exp. Psychol, , 1960, 59, 60-67.

Stevens, J. C., \& Marks, L. E. Cross-modality matching of brightness and loudness. Proc. Nat. Acad. Sci., 1965, 54, 407-411.

Stevens, S. S. Tactile vibration: Dynamics of sensory intensity. $J$. exp. Psychol., 1959a, 57, 210-218.

Stevens, S. S. Cross-modality validation of subjective scales for loudness, vibration, and electric shock. J. exp. Psychol., 1959b, 57, 201-209.

Stevens, S. S., \& Guirao, Miguelina. Loudness, reciprocality, and partition scales. J. Acoust. Soc. Amer, 1962, 34, 1466-1471.

Stevens, S. S., \& Guirao, Miguelina. Subjective scaling of length and area and the matching of length to loudness and brightness. J. exp. Psychol., 1963, 66, 177-186.

Stevens, S. S., \& Harris, Judith R. The scaling of subjective roughness and smoothness. J. exp. Psychol., 1962, 64, 489-494. Note

1. Supported in part by grants from the National Science Foundation and the National Institutes of Health (Laboratory of Psychophysics Report PPR-318). 